\title{
ARTICLE \\ Aging mice show impaired memory updating in the novel OUL updating paradigm
}

Janine L. Kwapis ${ }^{1,2}$, Yasaman Alaghband ${ }^{1}$, Ashley A. Keiser ${ }^{1}$, Tri N. Dong ${ }^{1}$, Christina M. Michael ${ }^{1}$, Diane Rhee ${ }^{1}$, Guanhua Shu ${ }^{1}$, Richard T. Dang ${ }^{1}$, Dina P. Matheos ${ }^{1}$ and Marcelo A. Wood ${ }^{1}$

Memories do not persist in a permanent, static state but instead must be dynamically modified in response to new information. Although new memory formation is typically studied in a laboratory setting, most real-world associations are modifications to existing memories, particularly in the aging, experienced brain. To date, the field has lacked a simple behavioral paradigm that can measure whether original and updated information is remembered in a single test session. To address this gap, we have developed a novel memory updating paradigm, called the Objects in Updated Locations (OUL) task that is capable of assessing memory updating in a non-stressful task that is appropriate for both young and old rodents. We first show that young mice successfully remember both the original memory and the updated information in OUL. Next, we demonstrate that intrahippocampal infusion of the protein synthesis inhibitor anisomycin disrupts both the updated information and the original memory at test, suggesting that memory updating in OUL engages the original memory. To verify this, we used the Arc CatFISH technique to show that the OUL update session reactivates a largely overlapping set of neurons as the original memory. Finally, using OUL, we show that memory updating is impaired in aging, 18-m.o. mice. Together, these results demonstrate that hippocampal memory updating is impaired with aging and establish that the OUL paradigm is an effective, sensitive method of assessing memory updating in rodents.

Neuropsychopharmacology (2020) 45:337-346; https://doi.org/10.1038/s41386-019-0438-0

\section{INTRODUCTION}

Memories need to be dynamically updated to incorporate the most relevant, recent information into storage. This ability to integrate new information into existing memory is critical to allow organisms to anticipate future outcomes and adapt to new situations. Most memories are not new associations but are alterations or additions (updates) to existing memories, particularly in the aging, experienced brain. Despite its fundamental importance, the mechanisms that support memory updating are largely uncharacterized and even less is understood about how dysregulation of these mechanisms might contribute to agerelated cognitive decline. Understanding how memories are modified in response to new information is therefore an important step toward improving memory across the lifespan.

Memories may be updated through a process termed "reconsolidation," in which retrieval triggers a period of lability that could allow new information to be incorporated into an existing, stable memory. The reconsolidation process consists of an initial destabilization phase marked by protein degradation followed by a restabilization phase characterized by protein synthesis [1-9]. Recent work has demonstrated that reconsolidation is only initiated when new information is presented at retrieval; when retrieval consists of only familiar information, the original memory remains stable and resistant to amnesic agents like protein synthesis inhibitors $[2,10-16]$. This suggests that new information triggers memory destabilization to allow for modification. Consistent with this, numerous studies now indicate that new information presented during retrieval can alter the content of memory $[2,3,11,17,18]$, the affective component of memory [19-22], produce persistent extinction [23-27], or even reorganize the memory at the circuit level [11, 28].

Although it is clear that reconsolidation-based updating procedures can modify memory content, the neural mechanisms that support this process are not well understood. Most of the work on reconsolidation-dependent memory updating is based on fear memories, which are robust and persistent, acquired rapidly, and can inform clinical treatments for anxiety disorders. Fear memories are limited in a number of ways, however, that make them less than ideal for studying memory updating. First, fear associations are exceptionally strong and difficult to modify, as observed with both boundary conditions [29-32] and their resistance to persistent extinction [33-36]. Second, as freezing is not a carefully timed behavior, it is difficult to determine whether a rodent's freezing reflects fear from the original memory or the updated information. Updates involving a shift in timing between a tone and shock, for example, cannot behaviorally distinguish the original information from the updated information; both procedures typically produce freezing throughout the presentation of the conditional stimulus $[10,11]$. Finally, fear conditioning is an inherently stressful aversive learning task that does not closely model the routine associations that reflect daily life and are commonly affected with normal aging. Here, to address these concerns, we aimed to develop a novel, non-stressful hippocampus-dependent memory updating paradigm based on the object

\footnotetext{
${ }^{1}$ Department of Neurobiology and Behavior, Center for the Neurobiology of Learning and Memory, University of California, Irvine, Irvine, CA 92697, USA Correspondence: Janine L. Kwapis (jlk855@psu.edu)

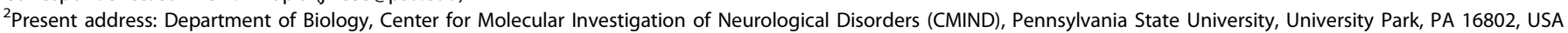

Received: 29 March 2019 Revised: 31 May 2019 Accepted: 7 June 2019

Published online: 15 June 2019 
location memory task, termed the Objects in Updated Locations (OUL) paradigm. The goals of this study were twofold. First, we aimed to establish the OUL task as a new method for studying hippocampus-dependent memory updating. Second, we aimed to use this paradigm to determine whether memory updating is impaired with age.

The OUL paradigm is novel in that it is able to assess both the original memory and the updated information in a single test session. Further, OUL uses incidental learning that takes advantage of rodents' innate preference for novelty, avoiding unnecessary stress and making it appropriate for testing age-related deficits in memory updating. Here, we first validate the OUL task, demonstrating that young mice show memory for both the original training and the memory update in the OUL test session. Next, we used two complementary methods (intra-hippocampal anisomycin injections and $\operatorname{Arc}$ (atFISH) to verify that the OUL update requires retrieval of the original memory, rather than forming a new, independent memory. Finally, using OUL, we demonstrate that aging mice show impairments in memory updating, suggesting that an inability to update memories may contribute to age-related cognitive decline. Together, these results demonstrate that the OUL paradigm can be used to understand the mechanisms underlying hippocampal memory updating across the lifespan.

\section{MATERIALS AND METHODS}

Mice

The subjects were young adult (2-4-months-old) or old (18-20 months old) male C57BL/6 J mice (Jackson Laboratory). Mice were housed, fed, and handled as described in the Supplemental Methods. All procedures were approved by the University of California, Irvine's Institutional Animal Care and Use Committee and were in compliance with the National Institutes of Health guidelines.

\section{OUL task}

Following handling and habituation (see Supplemental Methods), mice were trained with two identical objects in specific locations $\left(A_{1}\right.$ and $\left.A_{2}\right)$ for 1 or $3 d$ in the habituated context. Twenty four hours later, mice were given an update session, in which they were assigned to either the No Update condition or the Update condition. No Update mice were re-exposed to training locations $A_{1}$ and $A_{2}$. For Update mice, one object was moved to a new location $\left(A_{3}\right)$. Finally, mice were given a retention test in which they were exposed to the three objects in previously experienced locations $\left(A_{1}, A_{2}, A_{3}\right)$ and a fourth object in a novel location $A_{4}$. Memory for the original training formation was inferred by comparing exploration of the novel location $A_{4}$ to locations $A_{1}$ and $A_{2}$. Memory for the update was inferred by comparing exploration of the novel location $A_{4}$ to location $A_{3}$.

\section{Cannulation surgery}

Mice in Fig. 2 were implanted with chronic cannulae as previously described [37] to allow for direct hippocampal infusion of anisomycin after updating (see Supplemental Methods). Mice recovered for at least $7 \mathrm{~d}$ before behavioral testing began. Immediately after the update session, mice were bilaterally infused with anisomycin (ANI, $125 / \mu \mathrm{g} / \mu \mathrm{L}$ ) or vehicle (VEH) into the dorsal hippocampus $(1.0 \mu \mathrm{L} /$ side). Cannula placements were confirmed by staining coronal slices with cresyl violet.

\section{CatFISH}

Fluorescence in situ hybridization for CatFISH was performed as previously described [38, 39] (see Supplement for details). The DIG-labeled Arc antisense riboprobe was hybridized with tissue overnight and visualized with an anti-DIG-HRP conjugate, visualized with a Cy3 substrate kit, and counterstained with DAPI.
Confocal images were collected in area CA1b of the dorsal hippocampus and the images were scored to identify the subcompartmental localization of Arc to calculate a similarity score as previously described [39].

\section{Statistical analysis}

The OUL task was hand scored to measure object exploration times as previously described for object location memory experiments $[40,41]$. Statistical analyses (see Supplement) were performed using two-tailed Student's $t$-tests, one-way ANOVAs or two-way ANOVAs with Sidak-corrected $t$-tests. Mixed-model ANOVAs were used when one variable was a repeated measure. An a value of 0.05 was required for significance.

\section{RESULTS}

Young animals successfully show memory for both training and updating information in OUL

We first aimed to validate the OUL task as capable of assessing memory updating in young mice (Fig. 1a). Following habituation, mice were first trained to learn the locations of two identical objects in a familiar context (training session, Day 1). The following day, during the update session (Day 2), mice were split into two groups. The No Update group was re-exposed to the two familiar object locations $\left(A_{1}\right.$ and $\left.A_{2}\right)$. The Update group was exposed to one familiar object location $\left(A_{1}\right)$ and one object moved to a new location $\left(A_{3}\right)$. All groups were given a test session (on Day 3 ) to assess the animals' memory for both the original object locations and the updated location. At test, mice were exposed to four identical objects: three in previously exposed locations $\left(A_{1}, A_{2}\right.$, and $A_{3}$ ) and one in a novel location $\left(A_{4}\right)$. Memory for the original training was assessed by comparing exploration of the novel object location $\left(A_{4}\right)$ to exploration of the training object locations $\left(A_{1}\right.$ and $\left.A_{2}\right)$. Memory for the updated information was assessed by comparing exploration of the novel object location $\left(A_{4}\right)$ to exploration of the "updated" object location $\left(A_{3}\right)$. As mice prefer novelty, memory for either the original training session or the updated information is demonstrated by increased exploration of the object in the novel location $\left(A_{4}\right)$ compared to each of the other three objects (indicated by a higher score on the discrimination index (DI), see methods).

During training, mice in both groups showed similar levels of exploration of objects $A_{1}$ and $A_{2}$, resulting in a discrimination index (DI) near zero for both groups (Fig. 1 bi; two-tailed Student's $t$-test: $\left.t_{(17)}=0.086, p=0.40\right)$. Total exploration levels were also similar between the No Update and Update groups during training (Fig. 1bii; two-tailed Student's $t$-test: $t_{(17)}=0.035, p=0.97$, $n=10,9)$.

To confirm that the original object location memory was successfully acquired, we next assessed performance during the update session (Fig. 1C). Mice in the No Update group showed equal preference for the re-exposed locations $A_{1}$ and $A_{2}$, resulting in a DI near zero (Fig. $1 \mathrm{Ci}$ ). Mice in the Update group, on the other hand, preferentially explored the moved object $A_{3}$ compared to the unmoved object $A_{1}$ and showed a significantly higher DI compared to the No Update group (Fig. 1ci, two-tailed Student's $t$-test: $t_{(17)}=3.57, p=0.002$ ). Total exploration was also significantly higher in the Update group compared to the No Update group during the update session (Fig. 1cii; two-tailed Student's $t$-test: $t_{(17)}=3.39, p=$ 0.004), suggesting that mice spent more time exploring the objects when a novel location was introduced. Together, these results confirm that the original object locations were learned in young mice exposed to a 10-min training session, consistent with previous reports $[41,42]$.

To determine whether the original memory was modified to include the updated object location $\left(A_{3}\right)$, mice were given a test session in which each familiar location $\left(A_{1}, A_{2}\right.$, and $\left.A_{3}\right)$ was tested 
A

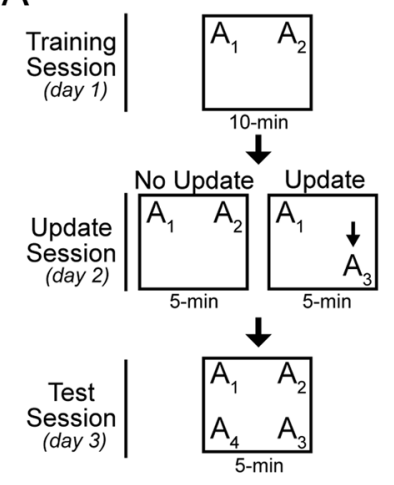

B

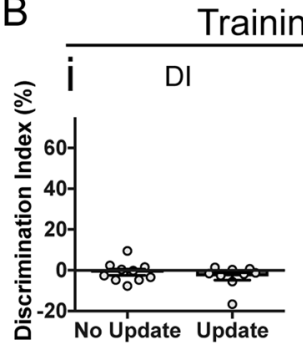

D
C

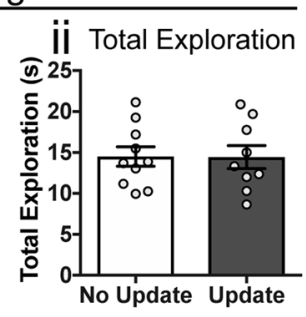

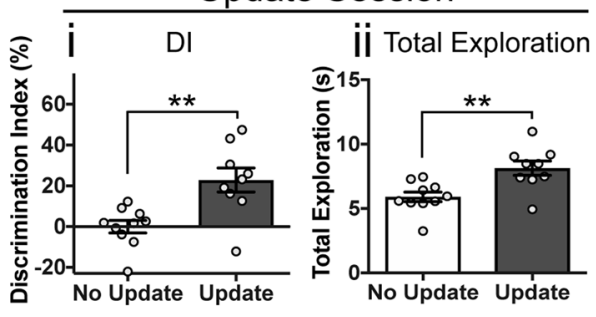

Test Session

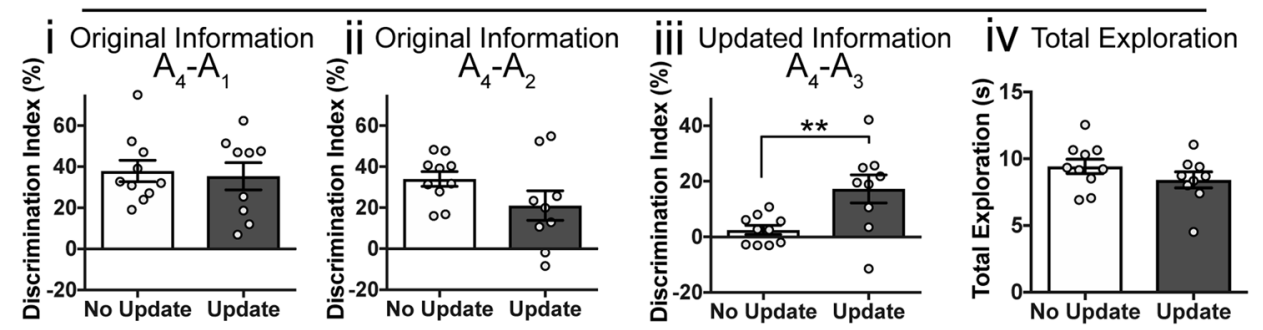

Fig. 1 Young mice successfully perform memory updating in the OUL task. a Experimental design. $\mathbf{b}$ Training session behavior. Mice show a low DI, indicating no preference for object $A_{1}$ or $A_{2}$ (left) and have similar levels of total object exploration (right). c Update session behavior. (i) Mice in the Update condition prefer the novel location $A_{3}$ to the familiar location $A_{1}$ whereas No Update mice show similar exploration of the familiar locations $A_{1}$ and $A_{2}$, with a DI near zero. (ii) Update mice show significantly more total object exploration than No Update mice. d Behavior during the test session. (i) Mice in both the Update and No Update group show intact memory for the original training object location $A_{1}$. (ii) Both groups also show memory for original training location $A_{2}$. (iii) Only the Update group shows a preference for the novel location $A_{4}$ over the updated location $A_{3}$; No Update mice prefer objects $A_{3}$ and $A_{4}$ equally. (iv) Mice show similar levels of total object exploration during the test session. Data are presented as mean $\pm \mathrm{SEM}$. ${ }^{* *} p<0.01$

against a novel object location $\left(A_{4}\right)$ (Fig. 1d). Raw percent exploration time for each of the four objects during the test session is shown in Supplementary Fig. 5A. Both groups showed intact memory for the original information, as both Update and No Update animals similarly preferred the novel location $\mathrm{A}_{4}$ over both original locations $A_{1}$ (Fig. 1di; two-tailed Student's $t$-test: $t_{(17)}=$ $0.31, p=0.76$ ) and $A_{2}$ (Fig. 1dii; two-tailed Student's $t$-test: $t_{(17)}=$ $1.66, p=0.12)$. This confirms that after updating, the original information is retained. To test whether the updated information was successfully learned, we also compared exploration of the novel location $A_{4}$ to the updated location $A_{3}$ during the test session. Mice in the No Update group showed equal preference for objects $A_{3}$ and $A_{4}$, resulting in a DI that was not significantly different from zero (Fig. 1diii; one-sample $t$-test compared to 0: $\left.t_{(9)}=1.55, p=0.156\right)$. Mice given the update, on the other hand, preferentially explored the novel location $\mathrm{A}_{4}$ over the updated location $A_{3}$ (Fig. 1diii, one-sample $t$-test compared to $0: t_{(8)}=3.42$, $p=0.009$; two-tailed Student's $t$-test comparing Update to No Update: $\left.t_{(17)}=2.92, p=0.0096\right)$, indicating that they recall the updated location. No differences in total exploration during the test session were observed between groups (Fig. 1div; two-tailed Student's $t$-test: $\left.t_{(17)}=1.27, p=0.22\right)$. Together, these results confirm that young animals exhibit preference behaviors consistent with successful recall of both the original information and the updated information at test.

Post-update hippocampal protein synthesis inhibition disrupts both the updated information and the original memory We next tested whether the updated information learned in the OUL task modifies the original memory or forms a new, independent association. For the OUL task to be a useful model of memory updating, the original memory needs to be retrieved and altered to incorporate the new object location information presented during the update. To test this, we used two complementary methods to examine whether the original memory is engaged by the OUL update session: anisomycin infusions and catFISH analysis.
First, we tested whether anisomycin applied after the update session would affect memory for the original training information. Numerous studies have demonstrated that post-retrieval infusion of the protein synthesis inhibitor anisomycin (ANI) can block reconsolidation under certain conditions [43, 44] at least temporarily [see 45]. Presumably, the original memory is made labile by the new information presented during retrieval but fails to properly restabilize in the absence of de novo protein synthesis, leading to a disruption of the original memory [6-9]. When no new information is presented during the retrieval trial, on the other hand, ANI infusions have no effect, as new information is required to initiate reconsolidation [6].

To determine whether the updated information in the OUL task engages the original memory, we infused anisomycin immediately after the update session and assessed whether this attenuated the original association (Fig. 2a). We infused ANI directly into the dorsal hippocampus $(\mathrm{DH})$ after the update session (Fig. 2b, individual cannula placements shown in Supplementary Fig. 1A), as spatial object location memories are particularly sensitive to manipulations in this region [40, 46, 47] and this would allow us to localize the memory update and avoid offtarget effects of global protein synthesis inhibition. Mice showed similar exploration of objects $A_{1}$ and $A_{2}$ at training (two-way ANOVA, no significant main effects or update $x$ drug interaction) and had similar levels of total exploration (two-way ANOVA, no significant main effects or update $x$ drug interaction) (Supplementary Fig. 1B, C). During the update session, mice in the Update condition showed a significant preference for the moved object, and mice in the No Update condition continued to show similar exploration of the objects in both familiar locations (Fig. 2ci; twoway ANOVA, significant main effect of update $\left(F_{(1,29)}=42.31, p<\right.$ $0.0001)$, but no significant effect of drug or significant interaction, $n=6,6,12,9)$ ). No group differences were observed between animals destined to get anisomycin or vehicle and no difference in total object exploration were observed during the update (Fig. 2cii; two-way ANOVA, no significant main effects or interaction). This suggests that before anisomycin infusion, mice 


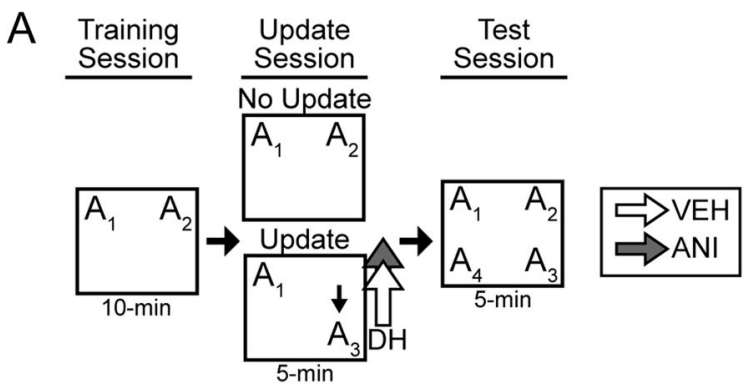

B

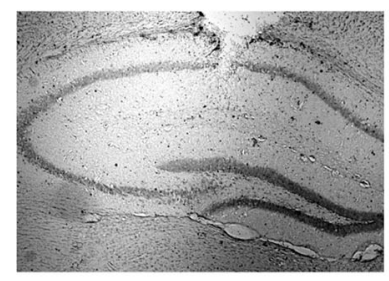

C

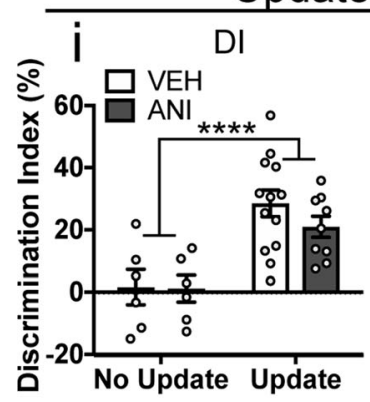

Fig. 2 Disrupting protein synthesis after memory updating impairs both the original memory and the updated information in young mice. a Experimental design. b Representative cannula placement targeting area CA1 of the dorsal hippocampus. c Update session behavior. (i) Both groups of mice in the Update condition preferred the novel location $A_{3}$ over the familiar location $A_{1}$ whereas No Update mice show a DI near zero, indicating no preference. (ii) Mice show similar total exploration across groups. d Behavior during the test session. (i) For original training object $A_{1}$, mice in the No Update condition show intact memory regardless of drug treatment. For the Update condition, anisomycin-treated mice show a significantly reduced $D I$ for $A_{1}$ compared to vehicle controls, indicating that anisomycin impaired memory for the original training information in Update animals. (ii) For original training object $A_{2}$, no significant effect of anisomycin was observed in either No Update or Update animals. (iii) For the updated location $A_{3}$, No Update animals showed a low DI compared to object $A_{4}$, regardless of drug infusion. Update animals given anisomycin showed a significantly lower DI than vehicle controls, indicating that anisomycin impaired memory for the updated information. iv) No significant differences in total exploration were observed at test. Data are presented as mean \pm SEM. ${ }^{* *} p<0.01,{ }^{* * *} p<0.001,{ }^{* * * *} p<0.0001$. VEH, vehicle; ANI, Aniosmycin

information was introduced, memory for both the original and the updated information was disrupted by anisomycin. To assess the original memory, we compared exploration of the object in a novel location $\left(A_{4}\right)$ to the objects in the training locations $A_{1}$ and $A_{2}$ (Fig. 2di, ii). Raw percent exploration time for each of the four objects during the test session is shown in Supplementary Fig. 5B. For object $A_{1}$, in the No Update group, mice in both drug conditions strongly preferred the novel location $A_{4}$ over the training location $A_{1}$ (Fig. 2di; two-way ANOVA, significant update by drug interaction $\left(F_{(1,29)}=4.66, p=0.039\right)$, Sidak's post hoc comparing No Update VEH vs ANI: $p=0.84, n=6,6)$. In the Update group, on the other hand, only vehicle animals preferred object $\mathrm{A}_{4}$; mice given anisomycin showed a significantly lower DI compared to vehicle controls (Sidak's post hoc comparing Update VEH vs ANI: $p=0.0004, n=12,9)$ indicating they had little preference for the object in a novel location. A similar pattern was observed for original object $A_{2}$ (Fig. 2dii), although there was no significant effect of anisomycin within either update condition (two-way ANOVA, significant effects of drug $\left(F_{(1,29)}=5.05, p=\right.$ $0.032)$ and update $\left(F_{(1,29)}=5.05, p=0.0324\right)$, but no drug $x$ update interaction, Sidak's post hocs comparing VEH vs ANI within each update condition, $p>0.05$ ), likely because object $A_{2}$ was not present for animals in the Update condition (see discussion). Together, these results suggest that anisomycin given after a memory update disrupts memory for the original training information.

We also assessed whether anisomycin disrupted memory for the updated information by comparing exploration of the novel location $A_{4}$ to the updated location $A_{3}$ (Fig. 2diii). Both vehicle and anisomycin mice in the No Update condition showed similar levels of exploration for objects $A_{3}$ and $A_{4}$, reflected as a DI near zero in both groups (two-way ANOVA, significant update by drug interaction $\left(F_{(1,29)}=6.17, p=0.02\right)$, Sidak's post hoc comparing No Update VEH vs ANI: $p=0.97$ ). For mice given the update, on the other hand, anisomycin impaired memory for the updated information; anisomycin animals showed significantly less preference for the novel location compared to vehicle animals (Sidak's post hoc comparing Update VEH vs ANI: $p=0.0003$ ). All groups showed similar levels of total exploration during the test session (Fig. 2div; twoway ANOVA, no significant main effects or interaction). Together, these results indicate that post-update anisomycin disrupts the updated information in addition to the information learned during training. successfully acquired long-term memory for the original training locations.

Immediately after the OUL update session, mice received intrahippocampal infusions of either anisomcyin or vehicle and memory was tested the following day. We found that when new 
The OUL update activates the neuronal ensemble supporting the original memory

To complement our anisomycin experiment, we also used the $\operatorname{Arc}$ catFISH technique to determine whether the update session reactivates the neuronal ensemble associated with the original memory or engages a new population of neurons in the dorsal hippocampus. CatFISH capitalizes on the temporal specificity of Arc mRNA localization following a learning event, using the subcellular distribution of $\operatorname{Arc}$ as an indication of the activity history of individual hippocampal neurons following two discrete events $[38,39,48]$.

We first verified that the timing of our experience-induced Arc mRNA expression matched that of previous reports, which observed intranuclear Arc mRNA foci within a few minutes after behavior and cytoplasmic Arc by 15-20 min after the event $[39,48,49]$. As this timing is critical to catFISH analysis, we first examined this expression pattern. Following a 10-min exposure to a novel context, mice were sacrificed 5,15 , or 30 min later and hippocampal slices were processed for Arc FISH (Supplementary Fig. 2A). Consistent with previous reports, we observed low background levels of Arc expression in our homecage controls (Supplementary Fig. 2Bi). For trained animals, five minutes after the training event, we saw predominantly punctate nuclear Arc mRNA expression whereas by $30 \mathrm{~min}$ after training, the staining was almost exclusively cytoplasmic (Supplementary Fig. 2Bii-iv). This timecourse of Arc expression matches that of numerous previous reports and confirms that the compartmentalization of Arc can be used to identify the neuronal ensemble activated during two discrete events.

Next, we used Arc catFISH to determine whether the neurons activated by the original training information would also be engaged by the updating event. Mice were first given three days of training to ensure robust learning of the original training information (Fig. 3a). Mice showed low DI scores throughout training, as mice explored objects $A_{1}$ and $A_{2}$ approximately equally (Supplementary Fig. $3 \mathrm{~A}, \mathrm{Bi}$, two-way mixed-model ANOVA, no significant main effect or day $x$ update interaction, but a significant effect of update type $\left(F_{(3,16)}=4.4, p=0.02\right)$ with no significant effect of any individual Sidak's post hoc test comparing updating conditions within each training day). No group differences were observed for total object exploration during training, although total object exploration did decrease across training days (Supplementary Fig. 3Bii, two-way mixed-model ANOVA, significant main effect of day $\left(F_{(2,32)}=18.32, p<0.0001\right)$, no significant effect of day or day $x$ update interaction). The following day (the "Update Sessions"), mice were given two discrete behavioral events at specific timepoints before sacrifice. For Epoch 1, all animals (except home cage controls) were reexposed to the training objects $A_{1}$ and $A_{2}$. No significant difference was observed between groups during the first epoch, with all groups showing approximately equal DI scores (Supplementary Fig. $3 \mathrm{Ci}$, left, $F_{(2,12)}=2.38, p=0.135$ ) and similar levels of object exploration (Supplementary Fig. 3Cii, left, $F_{(2,12)}=1.10, p=$ 0.364 ). Following a 20 -min rest in the colony room, mice were then given Epoch 2, in which they were either re-exposed to the training object locations (No Update/NU), were exposed to an object moved to a new location (Update/U), or were trained in a new context with new objects (New Memory/NM). During Epoch 2, mice in the Update group preferred the moved object and showed a significantly higher DI than No Update and New Memory Mice (Supplementary Fig. Ci, right, $F_{(2,12)}=8.09, p=$ 0.006 , Sidak's post hoc tests comparing $\mathrm{U}$ to $\mathrm{NU}, p=0.006$ and $\mathrm{U}$ to NM, $p=0.048$, NU to NM, $p=0.64$ ), demonstrating that the Update mice successfully remembered the training locations. The groups also showed different amounts of total object exploration during Epoch 2, with mice in the New Memory group showing significantly more object exploration than mice in either the No Update or Update group (Supplementary Fig. 3Cii, right,
$F_{(2,12)}=35.78, p<0.0001$, Sidak's post hoc tests comparing NM to $\mathrm{NU}, p<0.0001$, NM to $\mathrm{U}, p=0.0001$, NU to $\mathrm{U}, p=0.284$ ). This is consistent with numerous reports demonstrating that exposure to novel information (in this case, new objects) drives exploration in rodents [40, 50-52]. Mice were sacrificed immediately after the second epoch and CA1 hippocampal tissue was processed for catFISH. Cytoplasmic Arc expression was assumed to be activated by Epoch 1, whereas nuclear Arc was presumed to be activated by Epoch 2 (see representative images from this experiment, Fig. 3b), allowing us to calculate the relative overlap of the neuronal population activated by both events $[39,53]$.

We calculated a similarity score (see methods) for each of the behavioral conditions to quantify the ensemble overlap between the two epochs as previously described $[39,53]$. A high similarity score indicates that the same population of neurons was activated by both epochs whereas a low similarity score suggests that independent neuronal populations were activated by each event. We found that the Update condition produced approximately the same degree of ensemble overlap as the No Update condition (Fig. 3c, $n=5$ /group; one-way ANOVA, $F_{(2,12)}=18.36, p=0.0002$, Sidak's post hoc comparing NU to $U, p=0.26$ ) whereas the New Memory condition produced a significantly lower similarity score (Sidak's post hoc comparing NU to NM, $p=0.0002$; Sidak's post hoc comparing $U$ to $N M, p=0.003$ ), suggesting significantly less population overlap in the New Memory condition.

We also assessed the percent of cells expressing Arc in the cytoplasm, nucleus, or both across the behavioral conditions (Fig. 3d). The home cage group showed the lowest number of Arc positive cells at each of the subcellular distributions (cytoplasmic only, nuclear only, or both) (two-way ANOVA, significant update by compartment interaction $\left(F_{(6,32)}=26.32, p<0.0001\right)$, Sidak's post hocs comparing homecage to other behavioral conditions: cytoplasm-only, $p<0.001$, nuclear-only, $p<0.05$, both, $p<0.0001$, $n=5$ /group), establishing that we had low background staining of Arc. Consistent with the similarity score, we saw no significant difference between the No Update and the Update groups in the number of cells positive for both cytoplasmic and nuclear Arc staining ("Both"; Sidak's post hoc, $p=0.69$ ), suggesting that an approximately equivalent number of the "original memory" cells were reactivated during the OUL update session. In comparison, the New Memory group had significantly fewer cells positive for both cytoplasmic and nuclear Arc (Sidak's post hocs, both NU vs. $\mathrm{NM}, p=0.0002$, both $\mathrm{U}$ vs. NM, $p=0.006$ ), suggesting that more cells were uniquely activated by epochs 1 or 2 . Notably, the New Memory condition showed significantly more nucleus-only $\operatorname{Arc}$ staining compared to the No Update condition (Sidak's post hoc, nuclear-only NU vs NM, $p=0.021$ ) suggesting that novel information presented during epoch 2 might activate additional neurons. Overall, the proportion of CA1 cells expressing Arc in the current study was similar to that reported in previous studies $[48,53]$. Importantly, there was no difference in the total number of cells counted between the four behavioral conditions (Fig. 3e, one-way ANOVA, $\left.F_{(3,16)}=0.72, p=0.55\right)$. Together, these data suggest that the OUL update session reactivates a largely overlapping set of neurons that were engaged during original object location memory formation in area CA1 of the dorsal hippocampus. Together with the results of the anisomycin experiment, these data suggest that the OUL update session reactivates the original memory, rather than being encoded as a separate association.

Memory updating is impaired in aging mice

Finally, we used the OUL task to determine whether memory updating is impaired in aging mice. We trained young and old animals with three successive days of 10-min training to ensure successful acquisition of the original information (Fig. 4a), as we have previously shown that old (18-months-old) mice show deficits in long-term object location memory using a single $10 \mathrm{~min}$ 


\section{A}
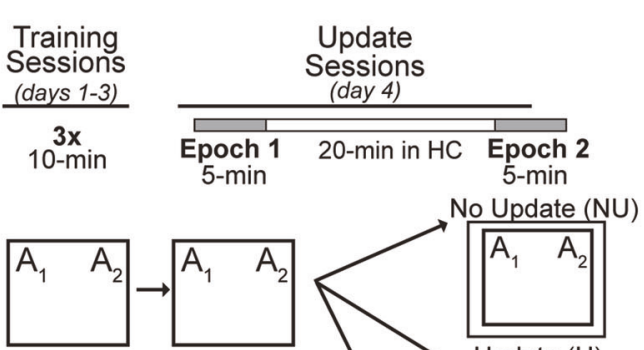

C

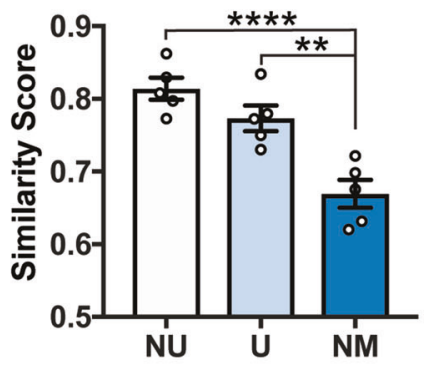

D
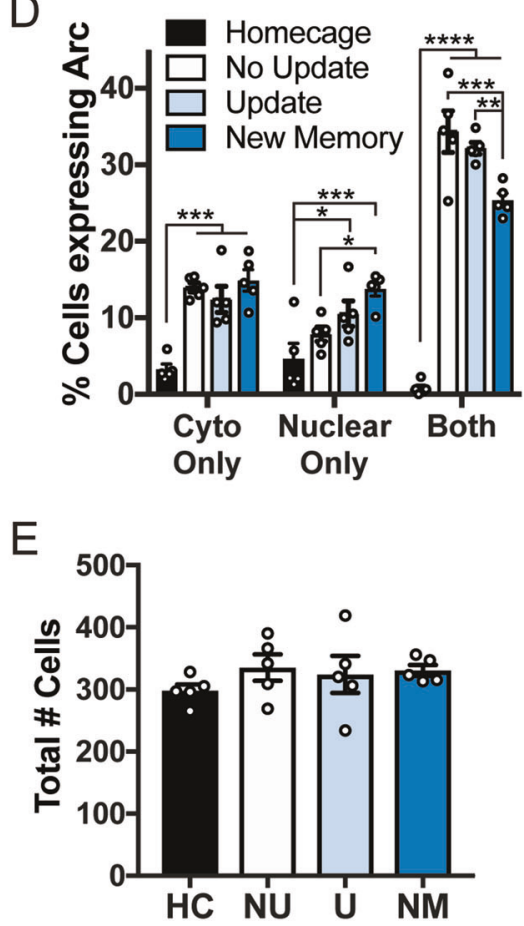

Fig. 3 The memory update activates the same degree of ensemble overlap in CA1 as re-exposure to the original training information. a Experimental design. On day 4 (Update Sessions), mice either remained in their homecages (HC) or were exposed to two discrete behavioral events (epochs 1 and 2) separated by 20 minutes. Mice were sacrificed immediately after Epoch 2. b Examples of hippocampal cells from animals in a that were counted as having (i) no Arc staining, (ii) nuclear Arc only (green arrow), (iii) cytoplasmic Arc only (yellow arrow), or (iv) both nuclear and cytoplasmic Arc (white arrow). Arc mRNA is red, DAPI nuclear counterstain is blue. c Ensemble overlap in the three behavioral conditions. There was no difference in the similarity scores between the No Update (NU) and the Update (U) groups whereas the New Memory (NM) group showed a significantly lower similarity score, indicating less ensemble overlap between epochs 1 and 2 than the other groups. d The percent of cells expressing Arc in each subcellular category. Homecage animals consistently showed less Arc expression than all other behavioral conditions. e A similar number of total cells were counted in each group. Data are presented as mean \pm SEM. ${ }^{*} p<0.05$, ${ }^{* *} p<0.01$, ${ }^{* * *} p<0.001,{ }^{* * * *} p<0.0001$

training period [41]. Mice were then given a standard 5-min update session on day 4 followed by the test session on day 5 .

Low DI scores were observed for both young and old mice throughout training, as mice explored objects $A_{1}$ and $A_{2}$ approximately equally (Supplementary Fig. 4, Bi, Bii, two-way mixed-model ANOVA, no significant main effects or day $x$ update interaction for young (i) or old (ii)). Similarly, no group differences for either young or old mice were observed for total object exploration across the three days of training, although total object exploration time did decrease across training days (Supplementary Fig. 4, iii, iv; two-way mixed-model ANOVA, significant main effect of day (Young: $F_{(2,28)}=17.87, p<0.0001$; Old: $F_{(2,26)}=10.51$, $p=0.0005)$, no significant effect of update or day $\mathrm{x}$ update interaction). To determine whether young and old mice successfully acquired memory following the three-day training protocol, we measured performance during the update session (day 4). Both young and old mice given the update showed intact memory for training, with both Update groups (i.e. groups exposed to the updated object location $A_{3}$ ) showing a significant preference for the moved object. No Update controls (i.e. mice reexposed to object locations $A_{1}$ and $A_{2}$ ) showed approximately equal preference for the two familiar locations (Fig. 4bi; two-way ANOVA comparing DIs, main effect of update $\left(F_{(1,29)}=51.08, p<\right.$ 0.0001 ), but no significant effect of age or age $x$ update interaction, Sidak's post hoc tests comparing No Update vs Update, young: $p<0.0001$, old: $p<0.0001, n=9,9,7,8$ ). Young animals given the update showed significantly more object exploration than their No Update controls (Fig. 4bii; two-way ANOVA, significant age or age $\times$ update interaction $\left(F_{(1,29)}=5.39\right.$, $p=0.028)$, Sidak's post hoc test comparing Young No Update vs Update, $p<0.0001$ ) whereas old animals showed similar total exploration time regardless of whether they were given the update (Sidak's post hoc test comparing Old No Update vs Update, $p=0.33$ ). This suggests that exposure to the novel location information drove increased exploration only in the young mice, possibly because aging reduces movement in general 

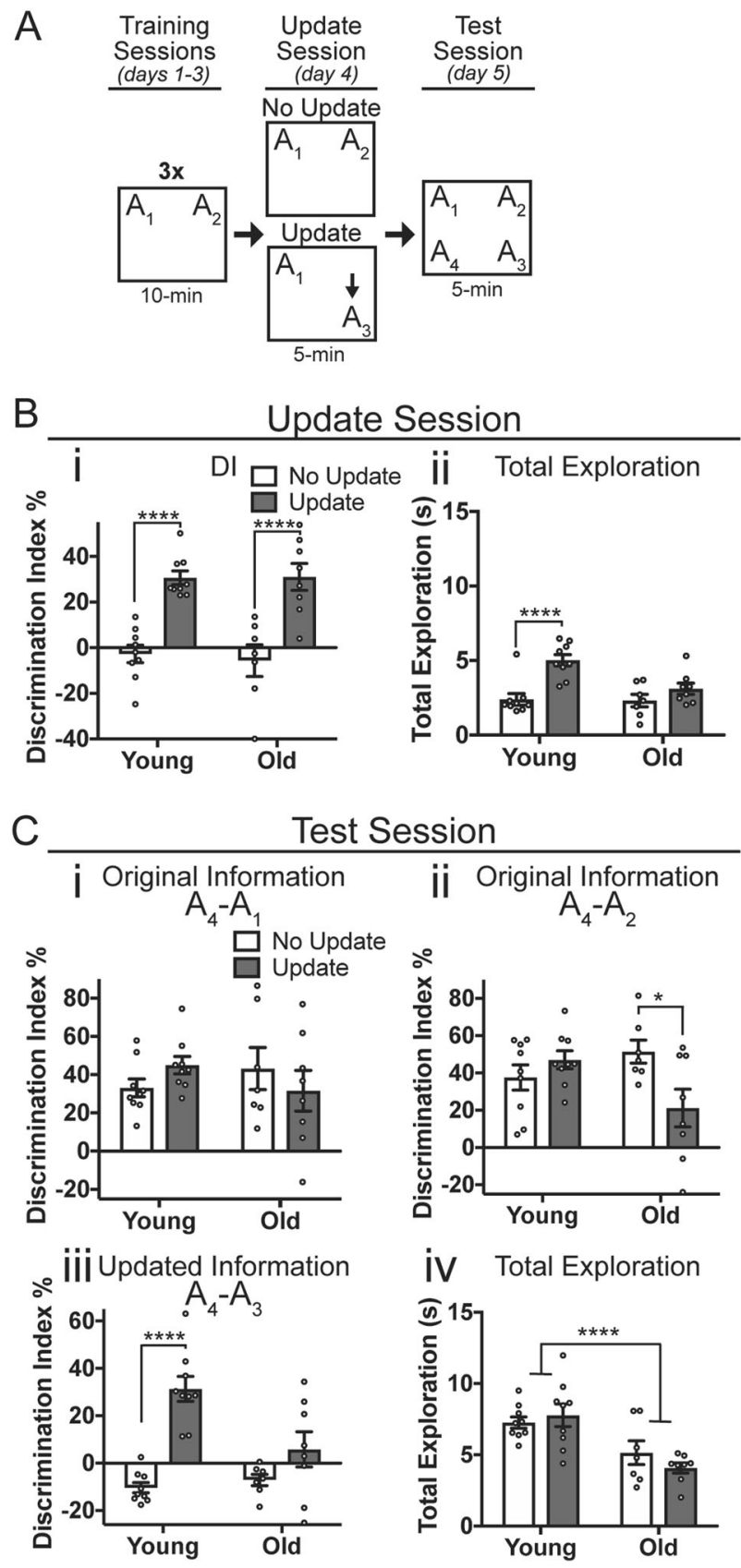

[e.g. 54]. Both young and old mice are therefore able to acquire the original object location memory with three successive days of 10-min training sessions.

Finally, we administered a retention test to determine whether the memory was successfully updated. Raw percent exploration time for each of the four objects during the test session is shown in Supplementary Fig. 5C. All animals showed intact memory for the original information, as young and old mice in both the Update and No Update conditions preferred the novel location $\mathrm{A}_{4}$ over the original location $A_{1}$ (Fig. 4ci; two-way ANOVA, no significant main effects or age $\times$ update interaction). Young mice also showed intact memory for the original object $A_{2}$ regardless of whether they received an update (Fig. 4cii; two-way ANOVA, significant age $\times$ update interaction $\left(F_{(1,29)}=7.61, p=0.01\right)$, Sidak's post hoc test comparing Young No Update vs. Update, $p=0.56$ ). In contrast, old mice given the update session showed a significantly lower $\mathrm{DI}$ for original object $A_{2}$ (Sidak's post hoc
Fig. 4 Aging mice show impairments in memory updating. a Experimental design. Young (3-months-old) and old (18-monthsold) mice were given three successive days of training before the day four update session. b Update session behavior. (i) Both young and old mice in the Update condition prefer the novel (updated) location $A_{3}$ over familiar location $A_{1}$. Mice in the No Update group show a low $\mathrm{Dl}$, indicating no preference for familiar locations $A_{1}$ or $A_{2}$. (ii) Young mice given the update show significantly more total exploration than young No Update mice. Old mice show similar exploration in the Update and No Update conditions. c Test session behavior. (i) All animals show intact memory for original training object $A_{1}$. (ii) For original training object $A_{2}$, young mice showed similar memory performance regardless of whether they were given the update. Old mice, in comparison, showed significantly lower DIs for object $A_{2}$ in the Update condition compared to the No Update condition. (iii) Young, but not old mice show intact memory for the updated location $A_{3}$. Young mice in the Update condition show a significantly higher DI $\left(A_{3}-A_{4}\right)$ compared to No Update controls. For old mice, both the No Update and Update groups show DIs near zero, indicating no preference for the novel location $A_{4}$ over the updated location $A_{3}$, even after updating. (iv) Total exploration was significantly lower in old animals compared to young, but no group differences were observed between Update and No Update groups. Data are presented as mean \pm SEM. ${ }^{*} p<0.05,{ }^{* * * *} p<0.0001$

comparing Old No Update vs. Update, $p=0.016$ ). This may reflect a weaker, less persistent memory for the original training information in aging mice, as object $A_{2}$ was not present during the update session for animals in the Update condition (see discussion).

To determine whether young and old mice successfully learned the memory update, we compared exploration of the updated location $A_{3}$ to the novel location $A_{4}$. Young mice given the update showed a significantly higher $\mathrm{DI}$ compared to their No Update controls (Fig. 4ciii; two-way ANOVA, significant age $x$ update interaction $\left(F_{(1,29)}=8.70, p=0.006\right)$, Sidak's post hoc test comparing Young No Update vs. Update, $p<0.0001$ ) indicating intact memory for the updated object $A_{3}$. In contrast, old mice showed little preference for the novel object regardless of whether they received the update session, with no significant difference in DI between old No Update and old Update mice (Sidak's post hoc test comparing Old No Update vs. Update, $p=0.16)$. Thus, although the 18 -monthsold mice were able to acquire the original memory, they showed an impaired ability to update this memory with the new object location. Overall, old mice explored less than young mice during the test session (Fig. 4civ; two-way ANOVA, significant effect of age $\left(F_{(1,29)}=21.72, p<0.0001\right)$, but no significant main effect of update or age $\times$ update interaction) although there were no differences between the Update and No Update group within each age condition. Correlating the amount of exploration during the training, updating, or test session with the test Dls for each object revealed no significant, meaningful relationship between total exploration time and test performance for either young or old animals, suggesting that reduced exploration in the aging mice cannot alone account for memory update performance. Thus, mice show age-related impairments in their ability to update a memory with new information in the OUL task.

\section{DISCUSSION}

Here, we developed and validated a novel OUL memory updating task, which can be used to understand the mechanisms supporting memory updating in young and old rodents. We show that young mice successfully perform OUL and show memory for both the original information and the updated information in a single test session. Next, using complementary approaches (catFISH and anisomycin application) we found that OUL updating engages the original memory, rather than driving the formation of an independent association. Finally, we used OUL to demonstrate 
that aging mice show deficits in memory updating. Together, these data indicate that age-related deficits in memory updating might contribute to age-related cognitive impairments. The OUL task is therefore a promising new tool to examine memory formation and updating considering its simplicity and reduced stress compared to water- and shock-based tasks. Most importantly, it allows for long-term memory and updated information to be discriminated within a single test session.

Using our newly established OUL paradigm, we found that aging, 18-month-old mice have difficulty updating existing memories, suggesting that memory becomes less flexible in old age. Agerelated memory deficits are well-documented across species $[41,55,56]$ and impairments in reversal learning $[57,58]$ and behavioral flexibility [59] are common in old age. In humans, hippocampus-dependent episodic memories are particularly sensitive to aging $[60,61]$, which may reflect either a reduced response to novelty in old age or an increased susceptibility to interference $[60,62]$. Here, we believe it is more likely that our 18-month-old mice show deficient hippocampal memory updating due to proactive interference, rather than an inability to detect the moved object as novel. The old mice show a clear preference for the object in a new location during the update session (Fig. 4b), demonstrating that they recognize the new location as novel. This information does not seem to be incorporated into the long-term memory, however, as they fail to show memory for the updated location the following day (Fig. 4ciii). This failure to learn the update may be due to proactive interference, in which the stored original memory obstructs the incorporation (or retrieval) of the new information presented during the update session. Indeed, it is common for aging individuals to have difficulty forgetting stored information even when it is no longer relevant [62]. Here, it seems that the original information persists in its original state in aging mice despite its reduced relevance in the face of the updated information.

Although reconsolidation has been studied consistently since its renewed interest in 2000 [8], only recently has it been appreciated as a process essential for memory updating. This study, with other recent work $[2,3,10,11,14,15,17,32,63,64]$, shows that new information must be presented during the retrieval session to trigger the reconsolidation process; when no new information was presented during the update session, memory was not affected by anisomycin (Fig. 2di, No Update group). Presumably, when only familiar information is presented during retrieval, destabilization mechanisms are not engaged and the original memory persists, stable even in the presence of protein synthesis inhibition $[3,17]$. Interestingly, using an object recognition task, Rossato and colleagues [64] have demonstrated that anisomycin infusions do not affect the original memory after a pseudo-reactivation trial in which two novel objects are presented without either of the original training objects. With the results of the current study, this suggests that the original memory is only made labile when one of the original training objects is present during the update session along with some new information about the other object's location or identity. It is likely that when only unfamiliar information is presented during the pseudo-retrieval session, a new memory is formed without the need to destabilize the original memory, which again persists in its original state. Thus, the reconsolidation process appears to allow existing memories to update in response to new information.

One interesting finding in the current study was that both young and old animals in the Update condition showed reduced memory for object location $A_{2}$ at test compared to animals in the No Update condition (e.g. Fig. 1dii). As Update animals were not exposed to $A_{2}$ during the update session (they only received locations $A_{1}$ and $A_{3}$ ), this increased preference for $A_{2}$ is likely due to the longer retention period ( $48 \mathrm{~h}$ ) between training and testing for this location. Indeed, previous studies have also observed increased exploration of an object presented less recently compared to one presented more recently [65] suggesting that length of time between exposure and testing can affect an object's subjective novelty or the strength of the animal's memory for that object. No Update animals, in comparison, received object location $A_{2}$ during the update session and therefore only had a 24 $h$ retention period for $A_{2}$. For our old mice, we found that memory for object $A_{2}$ was significantly reduced in Update animals compared to No Update animals (Fig. 4cii), suggesting that even with three successive days of training, the original memory begins to degrade by $48 \mathrm{~h}$ in the aging brain. Young animals showed robust memory for object $A_{2}$ even $48 \mathrm{~h}$ after exposure. Measuring animals' preference for location $A_{2}$ may therefore provide additional information about the persistence of the original memory in comparison to the more recently experienced location $A_{1}$. As OUL also has a temporal component, in which the animals learn that object $A_{3}$ is presented after objects $A_{1}$ and $A_{2}$, exploration of location $A_{2}$ might provide some information about the temporal aspect of memory updating. Understanding how time plays into memory updating is an important consideration that should be the focus of future work.

As most memories are modifications, rather than completely de novo memories, it is critical to understand how existing memories are modified at a molecular level. Although numerous studies have investigated the mechanisms supporting memory reconsolidation, much of this work is unable to distinguish between mechanisms supporting the original memory versus those that support the updated information. Our OUL updating paradigm is wellpositioned to identify mechanisms involved in memory updating, as it is able to assess both the original memory and the updated information in a single test session. Future work should determine whether key mechanisms, such as AMPA receptor subunit exchange [26, 27], protein degradation $[4,66,67]$, and synthesis of key proteins such as zif268 and BDNF [17] are required for memory updating and whether these mechanisms are impaired in the aging hippocampus. Likewise, it will be interesting to determine whether information interference produces an opposing effect on neuronal ensembles and molecular events.

In conclusion, we found that the OUL paradigm is a sensitive, hippocampus-dependent task capable of assessing both an original memory and updated information in a single test session. We also demonstrate that aging mice show deficits in memory updating, suggesting that an inability to update existing memories may contribute to age-related cognitive decline. The OUL task is therefore a powerful tool that can be used to understand the neural mechanisms that support memory updating in the young brain and to determine how these mechanisms are impaired in old age.

\section{FUNDING AND DISCLOSURE}

This research was supported by National Institutes of Health grants MH101491, AG051807, and AG050787 to MAW and National Institute on Aging (NIA) grants F32 AG052303 and K99 AG056596 to JLK. The authors declare no competing interests. The authors declare no competing interests.

\section{ACKNOWLEDGEMENTS}

We would like to thank Dr. John Guzowski for his assistance analyzing and interpreting catFISH data and Drs. Jennifer Czerniawski, and Terra White for their technical support in conducting the catFISH experiments. We would also like to thank Dr. Adeela Syed and the UC Irvine Optical Biology Center (OBC) for training and assistance collecting the confocal images.

\section{ADDITIONAL INFORMATION}

Supplementary information accompanies this paper at (https://doi.org/10.1038/ s41386-019-0438-0).

Publisher's note: Springer Nature remains neutral with regard to jurisdictional claims in published maps and institutional affiliations. 


\section{REFERENCES}

1. Jarome TJ, Werner CT, Kwapis JL, Helmstetter FJ. Activity dependent protein degradation is critical for the formation and stability of fear memory in the amygdala. PloS ONE. 2011;6:e24349.

2. Jarome TJ, Ferrara NC, Kwapis JL, Helmstetter FJ. Contextual information drives the reconsolidation-dependent updating of retrieved fear memories. Neuropsychopharmacol: Off Publ Am Coll Neuropsychopharmacol. 2015;40:3044-52.

3. Lee JL. Memory reconsolidation mediates the strengthening of memories by additional learning. Nat Neurosci. 2008;11:1264-6.

4. Lee SH, Choi JH, Lee N, Lee HR, Kim Jl, Yu NK, et al. Synaptic protein degradation underlies destabilization of retrieved fear memory. Science. 2008;319:1253-6.

5. Nader K, Hardt O. A single standard for memory: the case for reconsolidation. Nat Rev Neurosci. 2009;10:224-34.

6. Lee JLC, Nader K, Schiller D. An update on memory reconsolidation updating Trends Cogn Sci. 2017;21:531-45.

7. Jarome TJ, Kwapis JL, Werner CT, Parsons RG, Gafford GM, Helmstetter FJ. The timing of multiple retrieval events can alter GluR1 phosphorylation and the requirement for protein synthesis in fear memory reconsolidation. Learn Mem. 2012;19:300-6.

8. Nader K, Schafe GE, Le Doux JE. Fear memories require protein synthesis in the amygdala for reconsolidation after retrieval. Nature . 2000;406:722-6.

9. Parsons RG, Gafford GM, Baruch DE, Riedner BA, Helmstetter FJ. Long-term stability of fear memory depends on the synthesis of protein but not mRNA in the amygdala. Eur J Neurosci. 2006;23:1853-9.

10. Diaz-Mataix L, Ruiz Martinez RC, Schafe GE, LeDoux JE, Doyere V. Detection of a temporal error triggers reconsolidation of amygdala-dependent memories. Curr Biol. 2013;23:467-72.

11. Kwapis JL, Jarome TJ, Ferrara NC, Helmstetter FJ. Updating procedures can reorganize the neural circuit supporting a fear memory. Neuropsychopharmacol: Off Publ Am Coll Neuropsychopharmacol. 2017;42:1688-97.

12. Morris RG, Inglis J, Ainge JA, Olverman HJ, Tulloch J, Dudai Y, et al. Memory reconsolidation: sensitivity of spatial memory to inhibition of protein synthesis in dorsal hippocampus during encoding and retrieval. Neuron. 2006;50:479-89.

13. De Oliveira Alvares L, Crestani AP, Cassini LF, Haubrich J, Santana F, Quillfeldt JA. Reactivation enables memory updating, precision-keeping and strengthening: exploring the possible biological roles of reconsolidation. Neuroscience. 2013;244:42-8.

14. Sevenster $D$, Beckers $T$, Kindt $M$. Retrieval per se is not sufficient to trigger reconsolidation of human fear memory. Neurobiol Learn Mem. 2012;97:338-45.

15. Sevenster D, Beckers T, Kindt M. Prediction error governs pharmacologically induced amnesia for learned fear. Sci (New Y, NY). 2013;339:830-3.

16. Sevenster $D$, Beckers $T$, Kindt $M$. Prediction error demarcates the transition from retrieval, to reconsolidation, to new learning. Learn Mem (Cold Spring Harb, NY). 2014;21:580-4.

17. Lee JL. Memory reconsolidation mediates the updating of hippocampal memory content. Front Behav Neurosci. 2010;4:168.

18. Hupbach A, Gomez R, Hardt O, Nadel L. Reconsolidation of episodic memories: a subtle reminder triggers integration of new information. Learn Mem (Cold Spring Harb, NY). 2007;14:47-53.

19. Haubrich J, Crestani AP, Cassini LF, Santana F, Sierra RO, Alvares Lde O, et al. Reconsolidation allows fear memory to be updated to a less aversive level through the incorporation of appetitive information. Neuropsychopharmacol: Off Publ Am Coll Neuropsychopharmacol. 2015;40:315-26.

20. Goltseker K, Levi L, Barak S. Counterconditioning during reconsolidation prevents relapse of cocaine memories. Neuropsychopharmacology. 2016;41:716-26.

21. Zeng XX, Du J, Zhuang CQ, Zhang JH, Jia YL, Zheng XF. Unconditioned stimulus revaluation to promote conditioned fear extinction in the memory reconsolidation window. PloS ONE. 2014;9:e101589.

22. Cogan ES, Shapses MA, Robinson TE, Tronson NC Disrupting reconsolidation: memory erasure or blunting of emotional/motivational value? Neuropsychopharmacology. 2018;44:399-407.

23. Monfils MH, Cowansage KK, Klann E, LeDoux JE. Extinction-reconsolidation boundaries: key to persistent attenuation of fear memories. Science. 2009;324:951-5.

24. Graff J, Joseph NF, Horn ME, Samiei A, Meng J, Seo J, et al. Epigenetic priming of memory updating during reconsolidation to attenuate remote fear memories. Cell . 2014;156:261-76.

25. Schiller D, Monfils MH, Raio CM, Johnson DC, Ledoux JE, Phelps EA. Preventing the return of fear in humans using reconsolidation update mechanisms. Nature. 2010;463:49-53.

26. Clem RL, Huganir RL. Calcium-permeable AMPA receptor dynamics mediate fear memory erasure. Science. 2010;330:1108-12.

27. Rao-Ruiz P, Rotaru DC, van der Loo RJ, Mansvelder HD, Stiedl O, Smit AB, et al. Retrieval-specific endocytosis of GluA2-AMPARs underlies adaptive reconsolidation of contextual fear. Nat Neurosci. 2011;14:1302-8.
28. Winters BD, Tucci MC, Jacklin DL, Reid JM, Newsome J. On the dynamic nature of the engram: evidence for circuit-level reorganization of object memory traces following reactivation. J Neurosci. 2011;31:17719-28.

29. Eisenberg M, Kobilo T, Berman DE, Dudai Y. Stability of retrieved memory: inverse correlation with trace dominance. Sci (New Y, NY). 2003;301:1102-4.

30. Suzuki A, Josselyn SA, Frankland PW, Masushige S, Silva AJ, Kida S. Memory reconsolidation and extinction have distinct temporal and biochemical signatures. J Neurosci. 2004;24:4787-95.

31. Wang SH, de Oliveira Alvares L, Nader K. Cellular and systems mechanisms of memory strength as a constraint on auditory fear reconsolidation. Nat Neurosci. 2009;12:905-12.

32. Winters BD, Tucci MC, DaCosta-Furtado M. Older and stronger object memories are selectively destabilized by reactivation in the presence of new information. Learn Mem (Cold Spring Harb, NY). 2009;16:545-53.

33. Pavlov IP. Conditioned reflexes: an investigation of the physiological activity of the cerebral cortex. London: Oxford University Press; 1927.

34. Quirk GJ. Memory for extinction of conditioned fear is long-lasting and persists following spontaneous recovery. Learn Mem (Cold Spring Harb, NY). 2002;9:402-7.

35. Bouton ME, King DA. Contextual control of the extinction of conditioned fear: tests for the associative value of the context. J Exp Psychol Anim Behav Process. 1983;9:248-65.

36. Rescorla RA, Heth CD. Reinstatement of fear to an extinguished conditioned stimulus. J Exp Psychol Anim Behav Process. 1975;1:88-96.

37. Haettig J, Stefanko DP, Multani ML, Figueroa DX, McQuown SC, Wood MA. HDAC inhibition modulates hippocampus-dependent long-term memory for object location in a CBP-dependent manner. Learn Mem (Cold Spring Harb, NY). 2011;18:71-9.

38. Guzowski JF, Worley PF. Cellular compartment analysis of temporal activity by fluorescence in situ hybridization (catFISH). Curr Protoc Neurosci. 2001. Chapter 1: Unit 1.8.

39. Hartzell AL, Burke SN, Hoang LT, Lister JP, Rodriguez CN, Barnes CA. Transcription of the immediate-early gene Arc in CA1 of the hippocampus reveals activity differences along the proximodistal axis that are attenuated by advanced age. $J$ Neurosci. 2013;33:3424-33.

40. Vogel-Ciernia A, Wood MA. Examining object location and object recognition memory in mice. Curr Protoc Neurosci. 2014;69:1-17.

41. Kwapis JL, Alaghband Y, Kramar EA, Lopez AJ, Vogel Ciernia A, White AO, et al Epigenetic regulation of the circadian gene Per1 contributes to age-related changes in hippocampal memory. Nat Commun. 2018;9:3323.

42. Vogel-Ciernia A, Matheos DP, Barrett RM, Kramar EA, Azzawi S, Chen Y, et al. The neuron-specific chromatin regulatory subunit BAF53b is necessary for synaptic plasticity and memory. Nat Neurosci. 2013;16:552-61.

43. Tronson NC, Taylor JR. Molecular mechanisms of memory reconsolidation. Nat Rev Neurosci. 2007;8:262-75.

44. Duvarci S, Nader K. Characterization of fear memory reconsolidation. J Neurosci. 2004;24:9269-75.

45. Lattal KM, Abel T. Behavioral impairments caused by injections of the protein synthesis inhibitor anisomycin after contextual retrieval reverse with time. Proc Natl Acad Sci USA. 2004;101:4667-72.

46. Barrett RM, Malvaez M, Kramar E, Matheos DP, Arrizon A, Cabrera SM, et al. Hippocampal focal knockout of CBP affects specific histone modifications, longterm potentiation, and long-term memory. Neuropsychopharmacol: Off Publ Am Coll Neuropsychopharmacol. 2011;36:1545-56.

47. Haettig J, Sun Y, Wood MA, Xu X. Cell-type specific inactivation of hippocampal CA1 disrupts location-dependent object recognition in the mouse. Learn Mem (Cold Spring Harb, NY). 2013;20:139-46.

48. Guzowski JF, McNaughton BL, Barnes CA, Worley PF. Environment-specific expression of the immediate-early gene Arc in hippocampal neuronal ensembles. Nat Neurosci. 1999;2:1120-4.

49. Vazdarjanova A, McNaughton BL, Barnes CA, Worley PF, Guzowski JF. Experiencedependent coincident expression of the effector immediate-early genes arc and Homer 1a in hippocampal and neocortical neuronal networks. J Neurosci. 2002;22:10067-71.

50. Bardo MT, Bowsling SL, Robinet PM, Rowlett JK, Lacy M, Mattingly BA. Role of dopamine D1 and D2 receptors in novelty-maintained place preference. Exp Clin Psychopharmacol. 1993;1:101-09.

51. Barnett SA, Cowan PE. Activity, exploration, curiosity and fear: An ethological study. Interdiscip Sci Rev. 1976;1:43-62.

52. Ennaceur A, Delacour J. A new one-trial test for neurobiological studies of memory in rats. 1: behavioral data. Behav Brain Res. 1988;31:47-59.

53. Vazdarjanova A, Guzowski JF. Differences in hippocampal neuronal population responses to modifications of an environmental context: evidence for distinct, yet complementary, functions of CA3 and CA1 ensembles. J Neurosci. 2004;24:6489-96. 
54. Barreto G, Huang $\Pi$, Giffard RG. Age-related defects in sensorimotor activity, spatial learning, and memory in C57BL/6 mice. J Neurosurg Anesthesiol. 2010;22:214-9.

55. Burke SN, Barnes CA. Neural plasticity in the ageing brain. Nat Rev Neurosci. 2006;7:30-40.

56. Gallagher M, Rapp PR. The use of animal models to study the effects of aging on cognition. Annu Rev Psychol. 1997;48:339-70.

57. Schoenbaum G, Nugent S, Saddoris MP, Gallagher M. Teaching old rats new tricks: age-related impairments in olfactory reversal learning. Neurobiol Aging. 2002;23:555-64.

58. Weiler JA, Bellebaum C, Daum I. Aging affects acquisition and reversal of reward-based associative learning. Learn Mem (Cold Spring Harb, NY). 2008;15:190-7.

59. Beas BS, McQuail JA, Ban Uelos C, Setlow B, Bizon JL. Prefrontal cortical GABAergic signaling and impaired behavioral flexibility in aged F344 rats. Neuroscience . 2017;345:274-86.

60. Leal SL, Yassa MA. Neurocognitive aging and the hippocampus across species. Trends Neurosci. 2015;38:800-12.
61. Hedden T, Gabrieli JD. Insights into the ageing mind: a view from cognitive neuroscience. Nat Rev Neurosci. 2004;5:87-96.

62. Wilson IA, Gallagher M, Eichenbaum H, Tanila H. Neurocognitive aging: prior memories hinder new hippocampal encoding. Trends Neurosci. 2006;29:662-70.

63. Lee JL. Reconsolidation: maintaining memory relevance. Trends Neurosci. 2009;32:413-20.

64. Rossato Jl, Bevilaqua LR, Myskiw JC, Medina JH, Izquierdo I, Cammarota M. On the role of hippocampal protein synthesis in the consolidation and reconsolidation of object recognition memory. Learn Mem (Cold Spring Harb, NY). 2007;14:36-46.

65. Barker GR, Banks PJ, Scott H, Ralph GS, Mitrophanous KA, Wong LF, et al. Separate elements of episodic memory subserved by distinct hippocampal-prefrontal connections. Nat Neurosci. 2017;20:242-50.

66. Jarome TJ, Helmstetter FJ. The ubiquitin-proteasome system as a critical regulator of synaptic plasticity and long-term memory formation. Neurobiol Learn Mem. 2013;105:107-16.

67. Jarome TJ, Ferrara NC, Kwapis JL, Helmstetter FJ. CaMKII regulates proteasome phosphorylation and activity and promotes memory destabilization following retrieval. Neurobiol Learn Mem. 2016;128:103-9. 